\title{
Estado nutricional e sintomas gastrointestinais em adolescentes de uma capital do
}

\section{nordeste brasileiro}

\author{
Nutritional status and gastrointestinal symptoms in adolescents from a capital city in northeastern \\ Brazil
}

Estado nutricional y síntomas gastrointestinales en adolescentes de una ciudad capital del noreste

de Brasil

Recebido: 04/04/2021 | Revisado: 12/04/2021 | Aceito: 14/04/2021 | Publicado: 27/04/2021

\author{
Lisidna Almeida Cabral \\ ORCID: https://orcid.org/0000-0002-1622-9577 \\ Universidade Estadual do Ceará, Brasil \\ E-mail:lissidna@yahoo.com.br \\ Fernanda Maria Carvalho Fontenele \\ ORCID: http://orcid.org/0000-0003-0918-9340 \\ Universidade Estadual do Ceará, Brasil \\ E-mail:fernanda.fontenele@aluno.uece.br \\ Clarissa de Alencar Diógenes Rôla \\ ORCID: https://orcid.org/0000-0001-8589-9657 \\ Universidade Estadual do Ceará, Brasil \\ E-mail: clarissadio@yahoo.com \\ Noênia Alves de Araújo \\ ORCID: https://orcid.org/0000-0002-4048-4199 \\ Universidade Estadual do Ceará, Brasil \\ E-mail: noenianutri@gmail.com \\ Helena Alves de Carvalho Sampaio \\ ORCID: https://orcid.org/0000-0001-5353-8259 \\ Universidade Estadual do Ceará, Brasil \\ E-mail:dr.hard2@gmail.com \\ Francisco José Maia Pinto \\ ORCID: https://orcid.org/0000-0003-2976-7857 \\ Universidade Estadual do Ceará, Brasil \\ E-mail:maiapinto@yahoo.com.br
}

\begin{abstract}
Resumo
Objetivou-se correlacionar o estado nutricional com os sintomas gastrointestinais em adolescentes de uma capital do nordeste brasileiro. Trata-se de um estudo transversal com abordagem descritiva e analítica em Fortaleza/CE, no primeiro semestre de 2017 com 199 alunos de escolas públicas e privadas de 11 a 19 anos do $6^{\circ}$ ao $9^{\circ}$ ano. Os resultados mostraram que $66,8 \%$ tinha até 14 anos, $58,8 \%$ era do sexo feminino e 53,3\% oriunda de escola pública. 46,7\% adolescentes tinham excesso de peso, e 75,4\% apresentaram circunferência da cintura (CC) adequada. Embora a maioria esteja com a cintura com tamanho satisfatório, a razão cintura-estatura (RCE) esteve elevada em $59,8 \%$ estudantes. $35,2 \%$ dos adolescentes referiu sentir sempre ou quase sempre algum sintoma gastrointestinal, dos quais os mais frequentes foram: $12,6 \%$ gases, $12,1 \%$ distensão abdominal, $11,6 \%$ constipação e $10,1 \%$ dor. Houve relação significativa entre o estado nutricional e o estudante ser de escola pública ou privada, bem como entre a CC e a RCE com o excesso de peso.O sexo feminino tem maior risco de apresentar sintomas quando comparado ao sexo masculino. Adolescentes com CC elevada têm mais risco de desenvolver sintomas gastrintestinais. A associação entre sintomas e demais variáveis, só foi encontrada com o sexo no modelo ajustado. Diante dos resultados, percebe-se a necessidade da detecção precoce dos fatores de risco e mudanças no estilo de vida demonstrando forte associação da presença de sintomas gastrintestinais com o sexo feminino, e que o estado nutricional não apresentou associação com a presença de sintomas gastrointestinais.
\end{abstract}

Palavras-chave: Estado nutricional; Adolescente; Sinais e sintomas digestivos.

\section{Abstract}

The objective was to correlate nutritional status with gastrointestinal symptoms in adolescents from a capital city in northeastern Brazil. This is a cross-sectional study with a descriptive and analytical approach in Fortaleza / CE, which 
occurredin the first semester of 2017 with 199 students from public and private schools aged 11 to 19 years old, attending the 6th to 9 th grade. The results showed that $66.8 \%$ were up to 14 years old, $58.8 \%$ were female and $53.3 \%$ came from a public school. $46.7 \%$ of the adolescents were overweight, and $75.4 \%$ had adequate waist circumference (WC). Although most of them have a satisfactory waist size, the waist-to-height ratio (RCE) was high in $59.8 \%$ of the students. $35.2 \%$ of the adolescents reported feeling always or almost always some gastrointestinal symptom, of which the most frequent were: $(12.6 \%)$ gas, $(12.1 \%)$ abdominal distension, $(11.6 \%)$ constipation and $(10.1 \%)$ pain. There was a significant relationship between nutritional status and the type of school attended, as well as between WC and RCE with overweight. The female sex has a higher risk of presenting symptoms when compared to the male sex. Adolescents with elevated WC are more at risk of developing gastrointestinal symptoms. The association between symptoms and other variables was only found with sex in the adjusted model. In view of the results, there is a need for early detection of risk factors and changes in lifestyle, especially for females, which is strongly associated with the presence of gastrointestinal symptoms. Nutritional status was not associated with the presence of gastrointestinal symptoms.

Keywords: Nutritional status; Adolescent; Signs and symptoms, digestive.

\section{Resumen}

El objetivo fue correlacionar el estado nutricional con síntomas gastrointestinales en adolescentes de una ciudad capital en el noreste de Brasil. Se trata de un estudio transversal con enfoque descriptivo y analítico en Fortaleza / CE, en el primer semestre de 2017 con 199 alumnos de colegios públicos y privados de 11 a 19 años de $6^{\circ}$ a $9^{\circ}$ grado. Los resultados mostraron que el 66,8\% tenían hasta 14 años, el 58,8\% eran mujeres y el 53,3\% procedían de una escuela pública. El 46,7\% de los adolescentes tenía sobrepeso y el 75,4\% tenía una circunferencia de cintura (CC) adecuada. Aunque la mayoría tiene un tamaño de cintura satisfactorio, la relación cintura-altura (RCA) fue alta en el 59,8\% de los estudiantes. El 35,2\% de los adolescentes refirió presentar siempre o casi siempre algún síntoma gastrointestinal, de los cuales los más frecuentes fueron: (12,6\%) gases, $(12,1 \%)$ distensión abdominal, $(11,6 \%)$ estreñimiento y $(10,1 \%)$ dolor. Hubo una relación significativa entre el estado nutricional y el tipo de escuela a la que asistieron, así como entre CC y RCA con sobrepeso. El sexo femenino tiene mayor riesgo de presentar síntomas en comparación con el sexo masculino. Los adolescentes con CC elevada tienen un mayor riesgo de desarrollar síntomas gastrointestinales. La asociación entre síntomas y otras variables solo se encontró con el sexo en el modelo ajustado. A la vista de los resultados, existe la necesidad de una detección temprana de los factores de riesgo y cambios en el estilo de vida, especialmente para las mujeres, lo que tiene una fuerte asociación con la presencia de síntomas gastrointestinales. El estado nutricional no se asoció con la presencia de síntomas gastrointestinales.

Palabras clave: Estado nutricional; Adolescente; Signos y sintomas digestivos.

\section{Introdução}

Define-se por estado nutricional o grau de suprimento de nutrientes que atendam adequadamente às necessidades fisiológicas do organismo. Quando ocorre desequilíbrio nessa relação, podem ocorrer desordens nutricionais tanto pelo excesso quanto pela falta de nutrientes (WHO, 2000). O consumo alimentar elevado, com propensão à obesidade, pode determinar a ocorrência de enfermidades, como a resistência à insulina, síndrome metabólica, diabetes, hipertensão e dislipidemia, dentre outros, mesmo em crianças (Kilinc et al., 2019).

No último século, a obesidade infantojuvenil tornou-se um dos principais problemas de saúde. No Brasil, cerca de 3 milhões de jovens entre 13-17 anos apresentam excesso de peso (IBGE, 2016). Considerando a faixa etária de 12-17 anos, o sobrepeso e a obesidade atingem, respectivamente, 17,1\% e 8,4\% destas pessoas (Bloch et al., 2016).

Acompanhar o estado nutricional e a obesidade abdominal por meio de indicadores antropométricos é, portanto, imprescindível. A antropometria é uma técnica simples e de baixo custo, sendo muito utilizada para avaliar o risco de doenças associadas à obesidade, dada sua forte relação com a adiposidade corporal e visceral e com o risco cardiometabólico.

Algumas medidas antropométricas comumente utilizadas para esse fim são o índice de massa corporal (IMC), a circunferência da cintura $(\mathrm{CC})$ e a razão cintura-estatura $(\mathrm{RCE})$. A antropometria é importante para avaliar o estado nutricional 
na adolescência, permitindo monitorar a evolução e detectar déficits e excessos durante o crescimento (Kilinc et al., 2019;ABESO, 2016; De Oliveira et al., 2017).

Os distúrbios gastrointestinais, por sua vez, são definidos como condições crônicas do aparelho digestivo sem que uma causa seja detectada. Estas desordens são muito frequentes na prática clínica diária tanto na atenção primária quanto na prática gastroenterológica e atingem um importante segmento da população (Hunt et al., 2013). Os sintomas gastrointestinais frequentes, condicionam uma baixa qualidade de vida, sobretudo no público adolescente, acarretando alterações psicossociais importantes (Gonçalves, 2016). Além disso, esses sintomas podem ser precursores de problemas maiores, com alterações da barreira intestinal. Essas alterações estão associadas com distúrbios metabólicos, dentre eles o diabetes mellitus (Cheru et al., 2019), que por sua vez traz impactos negativos para o indivíduo, família e sociedade (Brutti et al., 2019). Sintomas intestinais também podem refletir diretamente na saúde mental e vice-versa (Ayonrinde et al., 2019).

Há evidências que fatores como alimentação, estilo de vida e regionalidade influenciam a microbiota intestinal, barreira intestinal e o sistema imunológico e, como consequência, as funções gastrointestinais podem ser alteradas em decorrência de hábitos alimentares irregulares (Garcia-Mantrana et al., 2018). Dentre as funções gastrointestinais alteradas em adolescentes decorrentes de seus hábitos alimentares, destacam-se constipação, diarreia, disbiose, náuseas e vômitos, gastrite e refluxo gastroesofágico (Ciampi et al., 2006).

Nesta perspectiva, os adolescentes merecem atenção especial por estarem em uma fase da vida crucial para a constituição de hábitos e valores importantes de vida saudável e que tendem a permanecer na vida adulta. Identificar os principais sintomas gastrointestinais e correlacionar com indicadores antropométricos é importante para delinear os grupos populacionais de risco para doenças associadas à obesidade. Com isso, podem ser planejados, operacionalizados e avaliados políticas, programas e intervenções relevantes na adolescência e que ajudem no controle das doenças crônicas da vida adulta.

O presente estudo objetivou, portanto, correlacionar o estado nutricional com os sintomas gastrointestinais em adolescentes de uma capital do nordeste brasileiro.

\section{Metodologia}

Trata-se de um estudo transversal com abordagem descritiva e analítica realizado (Padovani, 2012) em Fortaleza/CE, no primeiro semestre de 2017.

A pesquisa foi realizada em uma escola pública e uma privada de Fortaleza. Ambas foram escolhidas por conveniência, por concordarem com a pesquisa, mediante assinatura do termo de anuência.

Participaram da pesquisa adolescentes, de 11 a 19 anos, do $6^{\circ}$ ano do Ensino Fundamental ao $3^{\circ}$ ano do ensino médio. Foram excluídos os participantes que apresentavam alguma alteração neurológica que impossibilitasse responder às perguntas; os portadores de alguma doença no trato gastrointestinal, as grávidas e os ausentes no dia da coleta de dados.

Para o cálculo amostral, fez-se a estratificação por escola, considerando-se uma margem de erro de 5\%, intervalo de confiança de $95 \%$ e expectativa de prevalência de excesso de peso na população estudada em torno de $25 \%$. Participaram desta pesquisa, portanto, 199 estudantes, sendo 93 de escola particular e 106 de escola pública. A seleção dos estudantes ocorreu de forma aleatória, conforme a presença na lista de chamada, em cada umas das turmas, de cada colégio pesquisado.

Os dados foram coletados por acadêmicos de nutrição previamente treinados e supervisionados durante a coleta pelo responsável da pesquisa. Para transcrição dos dados, utilizou-se um formulário semiestruturado, contendo informações sobre: identificação (nome, data do nascimento, idade, escola), dados antropométricos (peso, altura, circunferência da cintura) e sintomas gastrointestinais, considerados como variável de desfecho dicotomizado (presença de sintomas; ausência de sintomas). 
A idade dos participantes foi agrupada utilizando-se a proposta por Rossi (2015) em que a adolescência é dividida em inicial (10 - 14 anos) e tardia (15 - 19 anos).

Para a coleta de peso, utilizou-se balança portátil da marca Omron, com capacidade para 150kg. A estatura foi aferida com estadiômetro portátil da marca Sanny. A partir desses dados, foi determinado o diagnóstico nutricional de acordo com as curvas do índice de massa corporal (IMC) para a idade, recomendadas pela World Health Organization em 2007 para crianças e adolescentes de 5 a 19 anos, e classificadas com base em Z-scores (WHO, 2007).

As categorias sobrepeso e obesidade foram agrupadas em excesso de peso. De forma semelhante, baixo peso e muito baixo peso foram agrupadas em baixo peso.

A circunferência da cintura foi aferida com o uso de fita métrica inelástica da marca Sanny, no ponto mínimo do tronco, conforme protocolo de Taylor et al. (2000). Foram considerados adequados os valores menores que o percentil 80.

A RCE, foi mensurada a partir do cálculo da razão entre a circunferência da cintura e a estatura em centímetros. Ela é um índice que possibilita indicar o acúmulo central de gordura e sua relação com a estatura de um indivíduo, a fim de minimizar avaliações equívocas quanto ao risco à saúde do indivíduo, controlando eventuais diferenças decorrentes da idade e/ou sexo, etnias e faixas etárias. O ponto de corte para classificação foi de 0,5 (Leone et al., 2014).

A coleta de dados referente à sintomatologia gastrointestinal foi realizada por meio do Questionário de Rastreamento Metabólico, adaptado do Instituto Brasileiro de Nutrição Funcional (IBNF). Foram considerados sintomas gastrointestinais: diarreia, distensão, constipação, gases, azia e dor abdominal.

Para a análise da variável desfecho, usou-se a frequência de sintomatologia dada pela escala de pontos, que varia de 0 a 4, em que 0,1, 2, 3 e 4 correspondem respectivamente a nunca, quase nunca, de vez em quando, quase sempre e sempre. Para fins de estatística, as frequências 'quase sempre' e 'sempre' foram agrupadas na categoria presença de sintomas, enquanto 'nunca', 'quase nunca' ou 'de vez em quando' foram agrupadas na categoria ausência de sintomas.

Para análise e tratamento dos dados, utilizou-se o programa Microsoft Office Excel®, versão 13.0 e o programa estatístico Statistical Package for Social Sciences (SPSS), versão 23.0.

$\mathrm{Na}$ análise descritiva utilizaram-se as frequências absolutas e percentuais. Na análise inferencial, foram verificadas as associações entre o desfecho e as variáveis explicativas por meio do teste Qui-quadrado Pearson. No modelo não ajustado, considerou-se o nível descritível p<0,20 como critério de entrada das variáveis para ajustamento do modelo. Somente foram consideradas no modelo multivariado as variáveis que apresentaram o valor de $\mathrm{p}<0,05$.

O presente estudo é resultado de um projeto maior, aprovado pelo Comitê de Ética em Pesquisa com Seres Humanos da instituição vinculada, sob parecer de número 1.993.052.

\section{Resultados}

Dos 199 adolescentes avaliados, verificou-se que a maioria $133(66,8 \%)$ tinha até 14 anos, $117(58,8 \%)$ era do sexo feminino e $106(53,3 \%)$ era oriunda de escola pública. Foram classificados como excesso de peso 93 (46,7\%) adolescentes, e $150(75,4 \%)$ com circunferência da cintura adequada. Embora a maioria esteja com a cintura com tamanho satisfatório, a razão cintura-estatura esteve elevada em 119 (59,8\%) estudantes. Quanto aos sintomas gastrointestinais, a maioria (70; 35,2\%) dosadolescentes referiu sentir sempre ou quase sempre algum sintoma, dos quais os mais frequentes foram: 25 (12,6\%) gases, $24(12,1 \%)$ distensão abdominal, $23(11,6 \%)$ constipação e $20(10,1 \%)$ dor (Tabela 1). 
Tabela 1. Características sociodemográficas, estado nutricional e presença de sintomas gastrointestinais em adolescentes do município de Fortaleza/CE, 2017.

\begin{tabular}{lrr}
\hline Variáveis & $\mathbf{n}$ & $\mathbf{\%}$ \\
\hline Sexo & 117 & \\
Feminino & 82 & 58,8 \\
Masculino & & 41,2 \\
Idade & 133 & \\
Até 14 anos & 66 & 66,8 \\
A partir de 15 anos & & 33,2 \\
Escola & 106 & \\
Pública & 93 & 53,3 \\
Privada & & 46,7
\end{tabular}

\section{Estado Nutricional}

Excesso de Peso 93

Eutrofia

Baixo Peso

\section{Circunferência da Cintura}

Adequada

Elevada

\section{Razão cintura-estatura}

Adequada

Elevada

Sintomas gastrointestinais com frequência

\begin{tabular}{lrr} 
Presentes & 70 & 35,2 \\
Ausentes & 129 & 64,8 \\
Sintomas Relatados & & \\
Diarreia & 3 & 1,5 \\
Constipação & 23 & 11,6 \\
Azia & 13 & 6,5 \\
Dor & 20 & 10,1 \\
Distensão Abdominal & 24 & 12,1 \\
Gases & 25 & 12,6 \\
\hline
\end{tabular}

Fonte: Autores (2021).

Ao se correlacionar o estado nutricional com as variáveis: sexo, idade, escola, RCE, cintura e sintomas gastrointestinais, verificou-se que houve diferença estatística $(\mathrm{p}<0,001)$, somente para as variáveis: escola, RCE e cintura (Tabela 2). 
Tabela 2. Correlação entre o estado nutricional e as variáveis relativas aos adolescentes de escola pública e privada de Fortaleza/CE, 2017.

\begin{tabular}{|c|c|c|c|c|c|c|c|}
\hline \multirow{3}{*}{ VARIÁVEIS } & \multicolumn{6}{|c|}{ Estado Nutricional } & \multirow[b]{3}{*}{$\mathbf{p}$} \\
\hline & \multicolumn{2}{|c|}{ Excesso de Peso } & \multicolumn{2}{|c|}{ Eutrofia } & \multicolumn{2}{|c|}{ Baixo Peso } & \\
\hline & $\mathbf{n}$ & $\%$ & $\mathbf{n}$ & $\%$ & $\mathbf{n}$ & $\%$ & \\
\hline \multicolumn{8}{|l|}{ Sexo } \\
\hline Masculino & 37 & 45,1 & 36 & 43,9 & 9 & 11 & 0,092 \\
\hline Feminino & 56 & 47,9 & 48 & 41 & 13 & 11,1 & \\
\hline \multicolumn{8}{|l|}{ Idade } \\
\hline Até 14 anos & 61 & 45,9 & 56 & 42,1 & 16 & 12 & 0,814 \\
\hline A partir de 15 anos & 32 & 48,5 & 28 & 42,4 & 6 & 9,1 & \\
\hline \multicolumn{8}{|l|}{ Escola } \\
\hline Pública & 32 & 30,2 & 59 & 55,7 & 15 & 14,2 & $<0,001$ \\
\hline Privada & 61 & 65,6 & 25 & 26,9 & 7 & 7,5 & \\
\hline \multicolumn{8}{|l|}{ RCE } \\
\hline Adequada & 9 & 11,3 & 53 & 66,3 & 18 & 22,5 & $<0,001$ \\
\hline Elevada & 84 & 70,6 & 31 & 26,1 & 4 & 3,4 & \\
\hline \multicolumn{8}{|l|}{ Cintura } \\
\hline Adequada & 49 & 32,7 & 82 & 54,7 & 19 & 12,7 & $<0,001$ \\
\hline Elevada & 44 & 89,8 & 2 & 4,1 & 3 & 6,1 & \\
\hline \multicolumn{8}{|l|}{ Sintomas } \\
\hline Presença & 40 & 57,1 & 25 & 35,7 & 5 & 7,1 & 0,079 \\
\hline Ausência & 53 & 41,1 & 59 & 45,7 & 17 & 13,2 & \\
\hline
\end{tabular}

Fonte: Autores (2021).

No modelo não ajustado foram selecionadas as variáveis: sexo $(\mathrm{p}=0,008)$, escola $(\mathrm{p}=0,061)$, cintura $(\mathrm{p}=0,047)$ e estado nutricional $(\mathrm{p}=0,079)$ (Tabela 3). 
Tabela 3. Correlação entre a presença de sintomas gastrointestinais e as variáveis do modelo não ajustado em adolescentes de escola pública e privada de Fortaleza/CE, 2017.

Sintomas gastrointestinais

\begin{tabular}{|c|c|c|c|c|c|c|c|}
\hline \multirow[t]{2}{*}{ VARIÁVEIS } & \multicolumn{2}{|c|}{ Presentes } & \multicolumn{2}{|c|}{ Ausentes } & \multirow[b]{2}{*}{$\mathbf{R P}$} & \multirow[b]{2}{*}{ IC } & \multirow[b]{2}{*}{$\mathbf{p}$} \\
\hline & $\mathbf{n}$ & $\%$ & $\mathbf{n}$ & $\%$ & & & \\
\hline \multicolumn{8}{|l|}{ Sexo } \\
\hline Masculino & 20 & 28,6 & 62 & 48,1 & 2,313 & $1,241-4,314$ & 0,008 \\
\hline Feminino & 50 & 71,4 & 67 & 51,9 & & & \\
\hline \multicolumn{8}{|l|}{ Idade } \\
\hline Até 14 anos & 47 & 67,1 & 86 & 66,7 & - & & 0,946 \\
\hline A partir de 15 anos & 23 & 32,9 & 43 & 33,3 & & & \\
\hline \multicolumn{8}{|l|}{ Escola } \\
\hline Privada & 39 & 55,7 & 54 & 41,9 & & & 0,061 \\
\hline Pública & 31 & 44,3 & 75 & 58,1 & & & \\
\hline \multicolumn{8}{|l|}{ Cintura } \\
\hline Adequada & 47 & 67,1 & 103 & 79,8 & 1,939 & $1,003-3,746$ & 0,047 \\
\hline Elevada & 23 & 32,9 & 26 & 20,2 & & & \\
\hline \multicolumn{8}{|l|}{ RCE } \\
\hline Adequada & 24 & 34,3 & 56 & 43,4 & - & & 0,210 \\
\hline Elevada & 46 & 65,7 & 73 & 56,6 & & & \\
\hline \multicolumn{8}{|l|}{ Estado Nutricional } \\
\hline Baixo Peso & 5 & 7,2 & 17 & 13,2 & - & & 0,079 \\
\hline Eutrofia & 25 & 35,7 & 49 & 45,7 & & & \\
\hline Excesso de Peso & 40 & 57,1 & 53 & 41,1 & & & \\
\hline
\end{tabular}

p: p-valor do Qui-quadrado de Pearson.

RP: Razão de Prevalência

IC: Intervalo de confiança $95 \%$

Fonte: Autores, (2021).

No modelo final ajustado de regressão múltipla, somente a variável sexo permaneceu com associação estatisticamente significante com a presença de sintomas gastrointestinais $(\mathrm{p}=0,006)$ (Tabela 4$)$.

Tabela 4. Análise de regressão de Poisson do modelo ajustado entre o desfecho e as variáveis, Fortaleza-CE, 2017.

\begin{tabular}{lrrr}
\hline Variáveis & RP & IC & p \\
\hline Masculino & 0,757 & $0,621-0,924$ & 0,006 \\
Feminino & 1,000 & & \\
\hline
\end{tabular}

p: p-valor referente ao Qui-quadrado de Pearson.

RP: Razão de Prevalência

IC: Intervalo de confiança $95 \%$

Fonte: Autores (2021).

\section{Discussão}

Os achados da pesquisa indicam relação significativa entre o estado nutricional e o estudante ser de escola pública ou privada. Também foi encontrado uma relação significativa da circunferência da cintura e da RCE com o excesso de peso, conforme esperado pelo acúmulo de gordura abdominal em pacientes com peso excessivo. Quanto aos sintomas gastrintestinais, o sexo feminino tem maior risco de apresentar sintomas quando comparado ao sexo masculino. Também foi identificado que adolescentes com circunferência da cintura elevada têm mais risco de desenvolver sintomas gastrintestinais. A 
associação entre sintomas e demais variáveis, só foi encontrada com o sexo feminino. Essa associação pode estar relacionada a problemas hormonais e emocionais.

Nesta pesquisa, a maioria dos adolescentes foi classificada na faixa de baixo peso a eutrófico. No entanto, a prevalência de adolescentes com sobrepeso e obesidade foi bem maior do que a encontrada por Nogueira et al. (2020), que foi de 29,6\%, e maior do que encontrada na Pesquisa Nacional de Saúde de 2019, em que 19,4\% dos adolescentes apresentam excesso de peso, conforme o IBGE (IBGE, 2020). Esse aspecto pode ser o reflexo do estilo de vida, condições socioeconômicas, ou região geográfica, próprias da região sudeste. Reconhece-se que existem diferenças socioeconômicas e culturais entre as regiões sudeste e nordeste.

O excesso de peso em adolescentes se tornou, nos últimos anos, um grave problema de saúde pública em diversos países de diferentes níveis econômicos (You \& Choo, 2016). A condição socioeconômica pode ser, portanto, um determinante importante desse aumento de prevalência de sobrepeso/obesidade nesse público, bem como do aumento do risco das doenças associadas.

A prevalência de excesso de peso entre estudantes da rede privada foi maior do que na rede pública, achados semelhantes aos de Silva et al. (Silva et al., 2018). O referido estudo avaliou a prevalência de sobrepeso e obesidade e seus fatores associados em escolares e adolescentes em uma cidade brasileira de médio porte, tendo como resultado o excesso de peso ou obesidade em 32,3\%, com prevalência maior nos estratos de maior condição econômica, bem como de maior escolaridade dos pais. Estes achados podem estar relacionados às famílias com estilo de vida mais sedentário e alto consumo de comidas hipercalóricas. Estudos que comparam o ambiente alimentar de escolas públicas e privadas no Brasil, destacam que nas escolas privadas o ambiente alimentar é mais obesogênico, com uma predominância de vendas de comida e bebidas ultraprocessadas (Carmo et al., 2018).

Neste estudo, a presença de adiposidade central foi investigada tanto com a CC como com a RCE. A CC esteve elevada em aproximadamente um quarto dos adolescentes, enquanto a RCE, em mais da metade. Como a RCE utiliza a CC como uma medida da adiposidade abdominal e a ajusta para o tamanho do indivíduo dividindo por sua altura, isso a torna mais sensível para detectar pacientes baixos com gordura abdominal não detectada na CC.

Não há muitos estudos na literatura que investiguem a presença de sintomas gastrointestinais em adolescentes e que estabeleçam sua relação com a antropometria. O mais comum é observar estudos em adultos. Nesse público, um estudo recente de Sezgin e colaboradores (Sezgin et al., 2019) verificaram a presença de sintomas em 70,6\% dos participantes, o que é aproximadamente o dobro do resultado observado no presente estudo. No entanto, vale ressaltar que eles classificaram como presença de sintoma qualquer outra frequência que não fosse "quase nunca". No presente estudo, foram considerados presentes apenas os sintomas com frequência 'quase sempre' ou 'sempre', o que justifica uma diferença grande de prevalência. A prevalência de sintomas do trato gastrointestinal superior, como azia, dor gástrica, náuseas, dentre outros, apresentou associação com o IMC elevado (sobrepeso e obesidade), o que não foi observado para sintomas referentes ao trato gastrointestinal inferior, como defecação anormal. Embora sem associação significante, foi observado que tanto os sintomas do trato superior como inferior, apresentaram prevalência aumentada com o aumento do IMC. Ogisu et al. (2020) também encontraram dados semelhantes, em que o IMC estava relacionado aos sintomas do trato gastrointestinal superior, refluxo e dispepsia.

Nesta pesquisa, o estado nutricional não apresentou relação significativa com os sintomas gastrointestinais. Porém, o parâmetro de CC apresentou significância estatística, mostrando que adolescentes com cintura abdominal elevada apresentam maiores riscos de ter sintomas gastrointestinais, o que evidencia a gordura abdominal como um fator importante para o desenvolvimento de tais sintomas. 
Essa relação entre a circunferência abdominal e os sintomas gastrointestinais foi avaliada por Bernal et al. (2013) em uma pesquisa realizada no México que contemplou mais de 1000 participantes, onde constatou significância estatística, mostrando que adolescentes com excesso de peso e circunferência abdominal elevada apresentam maiores riscos de terem sintomas principalmente, dispépticos, como azia, eructação e flatulência em comparação a pacientes eutróficos.

Embora alguns achados corroborem com a relação entre o estado nutricional e a circunferência da cintura com a prevalência aumentada de sintomas gastrointestinais, após a regressão de Poisson, essa hipótese não foi sustentada na presente pesquisa, mantendo-se apenas a associação com o sexo. Ogisu et al. (2020), cujo estudo já foi mencionado, além de constatarem a relação entre a antropometria básica e a prevalência, observaram que a presença de sintomas gastrointestinais superiores, como desconforto abdominal, saciedade precoce e empachamento, apresentaram diferença significativa entre os sexos, sendo maior em mulheres, o que é semelhante ao dado aqui encontrado.

As mulheres parecem apresentar mais sintomas gastrointestinais, podendo estar associado aos problemas hormonais e emocionais. A resposta está no envolvimento do sistema neuroendócrino que permite a comunicação bidirecional entre o intestino e o cérebro, proporcionando o surgimento de sintomas gastrointestinais (Del'Arco et al., 2017). Dados de outro estudo também encontraram prevalência maior de sintomas gastrointestinais em adolescentes do sexo feminino, assim como no presente estudo. As mulheres apresentaram 1,87 vezes a chance maior de desenvolver sintomas em comparação com o sexo masculino (Ayonrinde et al., 2019).

Dentre as limitações deste estudo, ressalta-se a possibilidade de existir uma causalidade reversa entre sintomas gastrointestinais e o estado nutricional, em virtude do delineamento do estudo ser transversal. Outro ponto a destacar é a limitação do tamanho da amostra não equitativa com relação ao sexo, bem como o fato de ter-se escolhido, por conveniência, apenas uma escola pública e privada. Em outras condições, poder-se-ia obter distintos resultados desta pesquisa.

Identificou-se, como lacuna desta pesquisa, a necessidade de investigar a relação entre estado nutricional e sintomas gastrointestinais com acréscimo da análise das variáveis sobre padrão alimentar e estado emocional. Sabe-se que pode haver associação com padrões alimentares distintos, bem como também a própria adaptação psicoemocional e social do adolescente. Esse parâmetro forneceria subsídios para analisar de maneira integral a ocorrência desses sintomas e sua associação com a nutrição ou outras causas.

\section{Conclusão}

Neste estudo, conclui-se que a presença de sintomas gastrintestinais tem forte associação com o sexo feminino. O estado nutricional não apresentou associação com a presença de sintomas gastrointestinais.

Sugere-se que o profissional de saúde seja capaz de identificar precocemente, fatores de risco e, por meio da educação em saúde, orientar o adolescente quanto aos cuidados para a manutenção da saúde e mudanças no estilo de vida. É condição sine qua non o acompanhamento periódico do indivíduo durante o ciclo de vida. $\mathrm{O}$ adolescente precisa ser incorporado na linha de cuidado de maneira a garantir uma assistência integral. Nessa perspectiva, há possibilidade do alcance da promoção da saúde nas fases subsequentes de sua vida.

Diante dos achados nesse estudo, destaca-se a importância e necessidade de futuros trabalhos nesse tema que é fundamental para compreender o adolescente e sua relação com a alimentação, estilo de vida e estado nutricional.

\section{Referências}

Associação Brasileira para o estudo da obesidade e da síndrome metabólica (ABESO). (2016). Diretrizes brasileiras de obesidade 2016. In VI Diretrizes Brasileiras de Obesidade (4th ed.). Associação Brasileira para o estudo da obesidade e da síndrome metabólica. https://doi.org/10.1590/S141552732000000100003 
Ayonrinde, O. T., Sanfilippo, F. M., O’Sullivan, T. A., Adams, L. A., Ayonrinde, O. A., Robinson, M., Oddy, W. H., \& Olynyk, J. K. (2019). Bowel patterns, gastrointestinal symptoms, and emotional well-being in adolescents: A cohort study. Journal of Gastroenterology and Hepatology (Australia), 34(11), 19461954. https://doi.org/10.1111/jgh.14699

Bernal-Reyes, R., López, A. M., \& Bernal-Serrano, M. (2013). Prevalencia de síntomas gastrointestinales en personas con sobrepeso y obesidad. Estudio epidemiológico en una población mexicana. Revista de Gastroenterología de México, 78(1), 28-34. https://doi.org/10.1016/j.rgmx.2012.10.006

Bloch, K. V., Klein, C. H., Szklo, M., Kuschnir, M. C. C., De Azevedo Abreu, G., Barufaldi, L. A., Da Veiga, G. V., Schaan, B., Da Silva, T. L. N., De Vasconcellos, M. T. L., Moraes, A. J. P., Borges, A. L., De Oliveira, A. M. A., Tavares, B. M., De Oliveira, C. L., De Freitas Cunha, C., Giannini, D. T., Belfort, D. R., Santos, E. L., \& Goldberg, T. B. L. (2016). ERICA: prevalências de hipertensão arterial e obesidade em adolescentes brasileiros. Rev Saúde Pública, 50(supl 1), 1s-12s. https://doi.org/10.1590/S01518-8787.2016050006685

Brutti, B., Flores, J., Hermes, J., Martelli, G., Porto, D. da S., \& Anversa, E. T. R. (2019). Diabete Mellitus: definição, diagnóstico, tratamento e mortalidade no Brasil, Rio Grande do Sul e Santa Maria, no período de 2010 a 2014. Brazilian Journal of Health Review, 2(4), 3174-3182. https://doi.org/10.34119/bjhrv2n4-083

Carmo, A. S. do, de Assis, M. M., Cunha, C. de F., de Oliveira, T. R. P. R., \& Mendes, L. L. (2018). The food environment of Brazilian public and private schools. Cadernos de Saude Publica, 34(12), 1-11. https://doi.org/10.1590/0102-311X00014918

Cheru, L., Saylor, C. F., \& Lo, J. (2019). Gastrointestinal Barrier Breakdown and Adipose Tissue Inflammation. Current Obesity Reports, 8, 165-174. https://doi.org/10.1007/s13679-019-00332-6

Ciampi, Il. A. Del, Galvão, L. C., \& Fernandes, M. I. (2006). Constipação intestinal: um termo desconhecido e distúrbio frequentemente não reconhecido. Revista Paulista de Pediatria, 24(2), 111-114.

Del'Arco, A. P. W. T., Magalhães, P., \& QuiliciUILICI, F. A. (2017). Sim Brasil Study - Women'S Gastrointestinal Health: Gastrointestinal Symptoms and Impact on the Brazilian Women Quality of Life. Arquivos de Gastroenterologia, 54(2), 115-122. https://doi.org/10.1590/s0004-2803.201700000-09

Garcia-Mantrana, I., Selma-Royo, M., Alcantara, C., \& Collado, M. C. (2018). Shifts on gut microbiota associated to mediterranean diet adherence and specific dietary intakes on general adult population. Frontiers in Microbiology, 9, 1-11. https://doi.org/10.3389/fmicb.2018.00890

Gonçalves, L. S. S. (2016). Qualidade de vida em crianças e adolescentes com sintomas de disfunção vesical e intestinal: uma revisão sistemática de estudos mistos (Issue June). Dissertação (Mestrado - Mestrado em Enfermagem) - Universidade de Brasília.

Hunt, R., Quigley, E., Abbas, Z., Eliakim, A., Emmanuel, A., Goh, K.-L., Guarner, F., Katelaris, P., Smout, A., Umar, M., Whorwell, P., Johanson, J., Saenz, R., Besançon, L., Ndjeuda, E., Horn, J., Hungin, P., Jones, R., Krabshuis, J., \& LeMair, A. (2013). Manejo dos sintomas comun s de doenças gastrointestinais na comunidade. In World Gastroenterology Organisation.

IBGE. (2020). Pesquisa Nacional de Saúde 2019: informações sobre domicílios, acesso e utilização dos serviços de saúde. In Instituto Brasileiro de Geografia e Estatistica- IBGE. IBGE, Coordenação de Trabalho e Rendimento. http://biblioteca.ibge.gov.br/visualizacao/livros/liv91110.pdf

IBGE - Instituto Brasileiro de Geografia e Estatística. (2016). Pesquisa nacional de saúde do escolar : 2015 . In Pesquisa nacional de saúde do escolar : 2015 / IBGE, Coordenação de População e Indicadores Sociais. IBGE.

Kilinc, A., Col, N., Demircioglu-Kilic, B., Aydin, N., Balat, A., \& Keskin, M. (2019). Waist to height ratio as a screening tool for identifying childhood obesity and associated factors. Pakistan Journal of Medical Sciences, 35(6), 1652-1658. https://doi.org/10.12669/pjms.35.6.748

Leone, C., Nascimento, V. G., Silva, J. P. C., \& Bertoli, C. J. (2014). Razão Cintura / Estatura: Marcador De Alteração Nutricional Em Pré-Escolares in Preschool Children ". Journal of Human Growth and Development, 24(3), 289-294.

Nogueira, L. R., de Mello Fontanelli, M., de Aguiar, B. S., Failla, M. A., Florindo, A. A., Leme, A. C., dos Anjos Souza Barbosa, J. P., \& Fisberg, R. M. (2020). Is the local food environment associated with excess body weight in adolescents in São Paulo, Brazil? Cad. Saúde Pública, 36(2), 1-15. https://doi.org/10.1590/0102-311X00048619

Ogisu, K., Masuda, A., Fujita, T., Yamazaki, Y., Kobayashi, M., Terao, S., Sanuki, T., Okada, A., Adachi, M., Arisaka, Y., Miyazaki, H., Yoshinaka, H., Kutsumi, H., Umegaki, E., \& Kodama, Y. (2020). Influence of sex on the association between body mass index and frequency of upper gastrointestinal symptoms. JGH Open, 4(5), 937-944. https://doi.org/10.1002/jgh3.12368

Oliveira, C. M., Ulbrich, A. Z., Neves, F. S., Dias, F. A. L., Horimoto, A. R. V. R., Krieger, J. E., Alvim, R. de O., \& Pere ira, A. D. C. (2017). Association between anthropometric indicators of adiposity and hypertension in a Brazilian population: Baependi Heart Study. PLoS ONE, 12(10), 1-10. https://doi.org/10.1371/journal.pone.0185225

Padovani, C. R. (2012). Bioestatistica. Cultura acadêmica: Universidade Estadual Paulista, Pró-Reitoria de Graduação.

Sezgin, O., Akpınar, H., Özer, B., Törüner, M., Bal, K., \& Bor, S. (2019). Population-based assessment of gastrointestinal symptoms and diseases: Cappadocia Cohort, Turkey. The Turkish Journal of Gastroenterology: The Official Journal of Turkish Society of Gastroenterology, 30(12), 1009-1020. https://doi.org/10.5152/tjg.2019.19882

Silva, A. P. da, Feilbelmann, T. C. M., Silva, D. C., Palhares, H. M. C., Scatena, L. M., Resende, E. A. M. R. de, \& Fátima B orges, M. de. (2018). Prevalence of overweight and obesity and associated factors in school children and adolescents in a medium-sized Brazilian city. Clinics (Sao Paulo, Brazil), 73 , e438. https://doi.org/10.6061/clinics/2018/e438

Taylor, R. W., Jones, I. E., Williams, S. M., \& Goulding, A. (2000). Evaluation of waist circumference, waist-to-hip ratio, and the conicity index as screening tools for high trunk fat mass, as measured by dual-energy X-ray absorptiometry, in children aged 3-19 y 13. American Journal of Clinical Nutrition, 72, 490495. https://doi.org/10.1093/ajcn/72.2.490

World Health Organization. (2000). Obesity: prevent - ing and managing the global epidemic. World Health Organization. 
Research, Society and Development, v. 10, n. 5, e6110514647, 2021

(CC BY 4.0) | ISSN 2525-3409 | DOI: http://dx.doi.org/10.33448/rsd-v10i5.14647

World Health Organization. (2007). Growth reference data for 5-19 years. WHO. https://www.who.int/tools/growth-reference-data-for-5to19-years

You, J., \& Choo, J. (2016). Adolescent overweight and obesity: Links to socioeconomic status and fruit and vegetable intakes. International Journal of Environmental Research and Public Health, 13, 1-11. https://doi.org/10.3390/ijerph13030307 